\title{
Usefulness of Intravitreal Bevacizumab for Retinopathy of Prematurity with Severely Dilated Tunica Vasculosa Lentis and Poor Mydriasis
}

\author{
Tomoaki Higashiyama Sanae Muraki Masahito Ohji \\ Department of Ophthalmology, Shiga University of Medical Science, Otsu, Japan
}

\section{Keywords}

Retinopathy of prematurity · Intravitreal bevacizumab - Laser therapy · Tunica vasculosa lentis · Mydriasis

\begin{abstract}
Background: Laser therapy has been the gold standard treatment for retinopathy of prematurity (ROP), while intravitreal bevacizumab (IVB) is reported to be of significant benefit for zone I ROP. A problem with laser therapy is that it is difficult to administer in ROP patients with severely dilated tunica vasculosa lentis and poor mydriasis. However, although IVB treatment has been performed in such severe ROP cases, only 1 report has discussed its usefulness. Case 1: A male infant was born with a birth weight of $382 \mathrm{~g}$ at 23 weeks' gestation. As visualization was poor and laser therapy could not be performed due to dilated tunica vasculosa lentis and poor mydriasis, IVB $(0.625 \mathrm{mg} / 0.025 \mathrm{~mL})$ was administered to both eyes. Following treatment, the ROP gradually improved, with regression of the dilated tunica vasculosa lentis and improvement of the mydriasis in both eyes. Case 2: A male infant was born with a birth weight of $698 \mathrm{~g}$ at 25 weeks' gestation. As laser therapy could not be performed due to severely dilated tunica vasculosa lentis and poor mydriasis, IVB $(0.625 \mathrm{mg} / 0.025 \mathrm{~mL})$ was administered to both eyes. Following treatment, the ROP gradually improved, with regression of the dilated tunica vasculosa lentis and improvement of the mydriasis in both
\end{abstract}




\section{Case Reports in Ophthalmology}

eyes. Conclusions: IVB is potentially more useful than laser therapy for the treatment of severe ROP with dilated tunica vasculosa lentis and poor mydriasis.

(c) 2017 The Author(s)

Published by S. Karger AG, Basel

\section{Introduction}

Retinopathy of prematurity (ROP) is a disorder of retinal vascular development and a major cause of childhood blindness [1]. Low birth weight and prematurity are strongly associated with the risk of ROP [2]. In cases of severe ROP, dilated tunica vasculosa lentis and poor mydriasis are evident, along with intravitreal angiogenesis [3-6]. Severe ROP, such as type 1 ROP defined by the Early Treatment for Retinopathy of Prematurity study, should be treated as soon as possible because it can rapidly progress to fibrovascular retinal detachment [7].

The current treatments for ROP are laser therapy and intravitreal bevacizumab (IVB) [8-11]. Laser therapy has been the gold standard treatment for ROP, while IVB is reported to be of significant benefit for zone I stage 3+ (i.e., stage 3 with plus disease) ROP when compared with laser therapy [8]. A problem with laser therapy is that it is difficult to administer in ROP patients with severely dilated tunica vasculosa lentis and poor mydriasis, because the treatment requires clear optic media and adequate mydriasis. However, IVB treatment can be used in such circumstances as it does not require clear optic media and adequate mydriasis.

To the best of our knowledge, only 1 report has described the usefulness of IVB for the treatment of severe ROP in which laser therapy was not feasible [12]. In this study, we report 2 cases in whom IVB was effective for the treatment of ROP with severely dilated tunica vasculosa lentis and poor mydriasis.

\section{Case Reports}

Case 1

A male infant was born with a birth weight of $382 \mathrm{~g}$ at 23 weeks' gestation. From birth, he received oxygen therapy for respiratory distress syndrome. The first ophthalmic examination was performed at 35.4 weeks because his general health had been poor. In the examination, type 1 ROP (zone I stage $3+$ ) with dilated tunica vasculosa lentis and poor mydriasis was diagnosed in both eyes (Fig. 1a, b, Fig. 2a, b). As laser therapy could not be performed due to dilated tunica vasculosa lentis and poor mydriasis, IVB $(0.625 \mathrm{mg} / 0.025 \mathrm{~mL})$ was administered to both eyes on the day following the first examination. Following treatment, the ROP gradually improved, with regression of the dilated tunica vasculosa lentis and improvement of the mydriasis in both eyes (Fig. 1c, d, Fig. 2c, d). Fundus examination 8 months after treatment revealed vascularization to the edge of the ora serrata, and a further fundus examination 1 year after treatment revealed no recurrence of the ROP.

Case 2

A male infant was born in a hospital with a birth weight of $698 \mathrm{~g}$ at 25 weeks' gestation. From birth, he received oxygen therapy for respiratory distress syndrome at the hospital. Ophthalmic examination revealed type 1 ROP (zone I stage $3+$ ) with dilated tunica vasculosa lentis and poor mydriasis in both eyes. At 37.4 weeks of age, he was transferred to our hospi- 
tal for treatment with IVB because laser therapy could not be performed due to severely dilated tunica vasculosa lentis and poor mydriasis. We performed a fundus examination and confirmed the diagnosis of type 1 ROP (zone I stage $3+$ ) with dilated tunica vasculosa lentis and poor mydriasis in both eyes (Fig. 3a, b). Treatment with IVB $(0.625 \mathrm{mg} / 0.025 \mathrm{~mL})$ was subsequently administered to both eyes immediately following the examination. Following treatment, the ROP gradually improved, with regression of the dilated tunica vasculosa lentis and improvement of the mydriasis in both eyes (Fig. 3c, d). Fundus examination 1 year after treatment revealed no recurrence of the ROP.

\section{Discussion}

We report 2 cases of ROP with severely dilated tunica vasculosa lentis and poor mydriasis who were both effectively treated with IVB. An advantage of IVB is that it can be performed in cases of severe ROP with poor visualization due to dilated tunica vasculosa lentis and poor mydriasis, when laser therapy is not feasible. Law et al. [12] reported that administration of IVB prior to laser therapy or vitrectomy improved treatment outcomes for ROP with poor mydriasis, iris rubeosis, and vitreous hemorrhage. In our current report, IVB was found to be effective as a monotherapy for severe ROP for which laser therapy could not be performed. Therefore, IVB is potentially more useful than laser therapy for the treatment of severe ROP with dilated tunica vasculosa lentis and poor mydriasis. In addition, such ROP cases might improve with only monotherapy of IVB.

IVB also offers other advantages for the treatment of ROP when compared with laser therapy. IVB can be used to treat ROP with dense vitreous hemorrhages and hazy media that make laser therapy difficult. The IVB procedure is also less invasive and takes less time than laser therapy. Finally, intravitreal injection is technically easier to perform than laser therapy, although there is a risk of complications, such as lens injury [8].

A previous study reported the efficacy of IVB for severe ROP. Mintz-Hittner et al. [8] reported significantly lower recurrence percentages of ROP with IVB treatment of zone I stage $3+$ ROP patients compared with laser therapy. Our current report also showed improvement in 2 cases of zone I stage 3+ ROP, with no recurrence following IVB treatment.

In conclusion, IVB has an advantage compared with laser therapy for ROP. IVB is potentially more useful than laser therapy for cases of severe ROP with dilated tunica vasculosa lentis and poor mydriasis.

\section{Statement of Ethics}

This study was approved by the Institutional Review Board of Shiga University of Medical Science and was conducted in accordance with the tenets of the Declaration of Helsinki. Written informed consent was obtained from the parents of the patients.

\section{Disclosure Statement}

The authors have no financial interest in any aspect of this report. 


\section{Case Reports in Ophthalmology}

References

1 Gilbert C: Retinopathy of prematurity: a global perspective of the epidemics, population of babies at risk and implications for control. Early Hum Dev 2008;84:77-82.

-2 Lad EM, Hernandez-Boussard T, Morton JM, Moshfeghi DM: Incidence of retinopathy of prematurity in the United States: 1997 through 2005. Am J Ophthalmol 2009;148:451-458.

-3 Schulenburg WE, Prendiville A, Ohri R: Natural history of retinopathy of prematurity. Br J Ophthalmol 1987;71:837-843.

-4 Axer-Siegel R, Snir M, Ron Y, Friling R, Sirota L, Weinberger D: Intravitreal bevacizumab as supplemental treatment or monotherapy for severe retinopathy of prematurity. Retina 2011;31:1239_ 1247.

-5 Chavala SH, Farsiu S, Maldonado R, Wallace DK, Freedman SF, Toth CA: Insights into advanced retinopathy of prematurity using handheld spectral domain optical coherence tomography imaging Ophthalmology 2009;116:2448-2456.

6 Sanghi G, Dogra MR, Katoch D, Gupta A: Aggressive posterior retinopathy of prematurity: risk factors for retinal detachment despite confluent laser photocoagulation. Am J Ophthalmol 2013;155:159-164. Hellström A, Smith LE, Dammann O: Retinopathy of prematurity. Lancet 2013;382:1445-1457.

Mintz-Hittner HA, Kennedy KA, Chuang AZ; BEAT-ROP Cooperative Group: Efficacy of intravitreal bevacizumab for stage 3+ retinopathy of prematurity. N Engl J Med 2011;364:603-615. Kuniyoshi K, Sugioka K, Sakuramoto H, Kusaka S, Wada N, Shimomura Y: Intravitreal injection of bevacizumab for retinopathy of prematurity. Jpn J Ophthalmol 2014;58:237-243.

10 Yetik H, Gunay M, Sirop S, Salihoglu Z: Intravitreal bevacizumab monotherapy for type-1 prethreshold, threshold, and aggressive posterior retinopathy of prematurity -27 month follow-up results from Turkey. Graefes Arch Clin Exp Ophthalmol 2015;253:1677-1683.

11 Hwang CK, Hubbard GB, Hutchinson AK, Lambert SR: Outcomes after intravitreal bevacizumab versus laser photocoagulation for retinopathy of prematurity: a 5-year retrospective analysis. Ophthalmology 2015;122:1008-1015.

12 Law JC, Recchia FM, Morrison DG, Donahue SP, Estes RL: Intravitreal bevacizumab as adjunctive treatment for retinopathy of prematurity. J AAPOS 2010;14:6-10. 


\section{Case Reports in Ophthalmology}
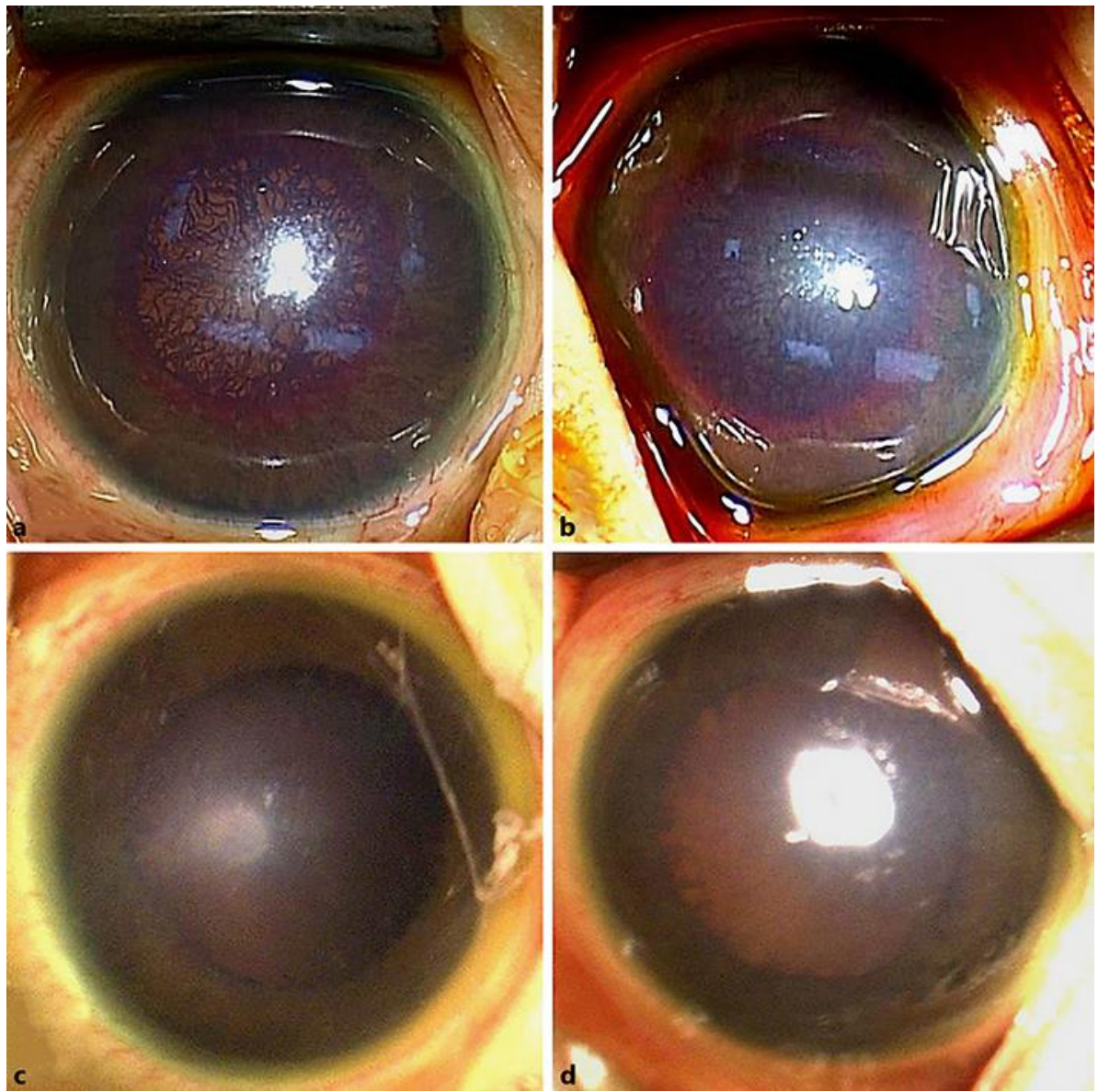

Fig. 1. Photographs of the anterior segment in case 1. a, b The anterior segment of the right eye (a) and the left eye (b) before treatment. Dilated tunica vasculosa lentis and poor mydriasis were observed in both eyes. c, $\mathbf{d}$ The anterior segment of the right eye (c) and the left eye (d) 4 days after treatment. The dilated tunica vasculosa lentis had regressed and the mydriasis was improved in both eyes. 


\section{Case Reports in Ophthalmology}
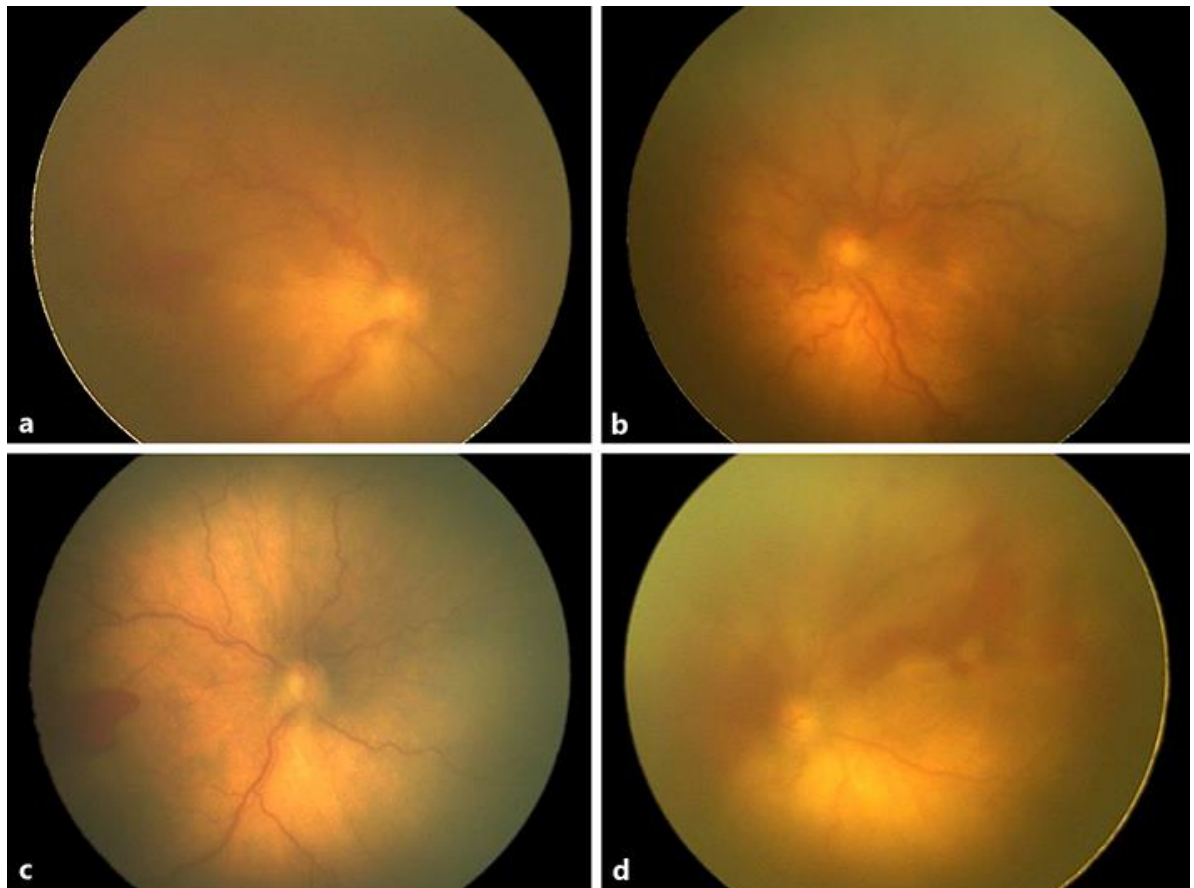

Fig. 2. Fundus photographs in case 1. a, b Fundus of the right eye (a) and the left eye (b) before treatment. Type 1 retinopathy of prematurity (ROP) with plus disease was observed in both eyes, although visualization was poor due to dilated tunica vasculosa lentis and poor mydriasis. $\mathbf{c}, \mathbf{d}$ The fundus of the right eye (c) and the left eye (d) 4 days after treatment. ROP was improved in both eyes although mild vitreous hemorrhage was observed after treatment. 


\section{Case Reports in Ophthalmology}
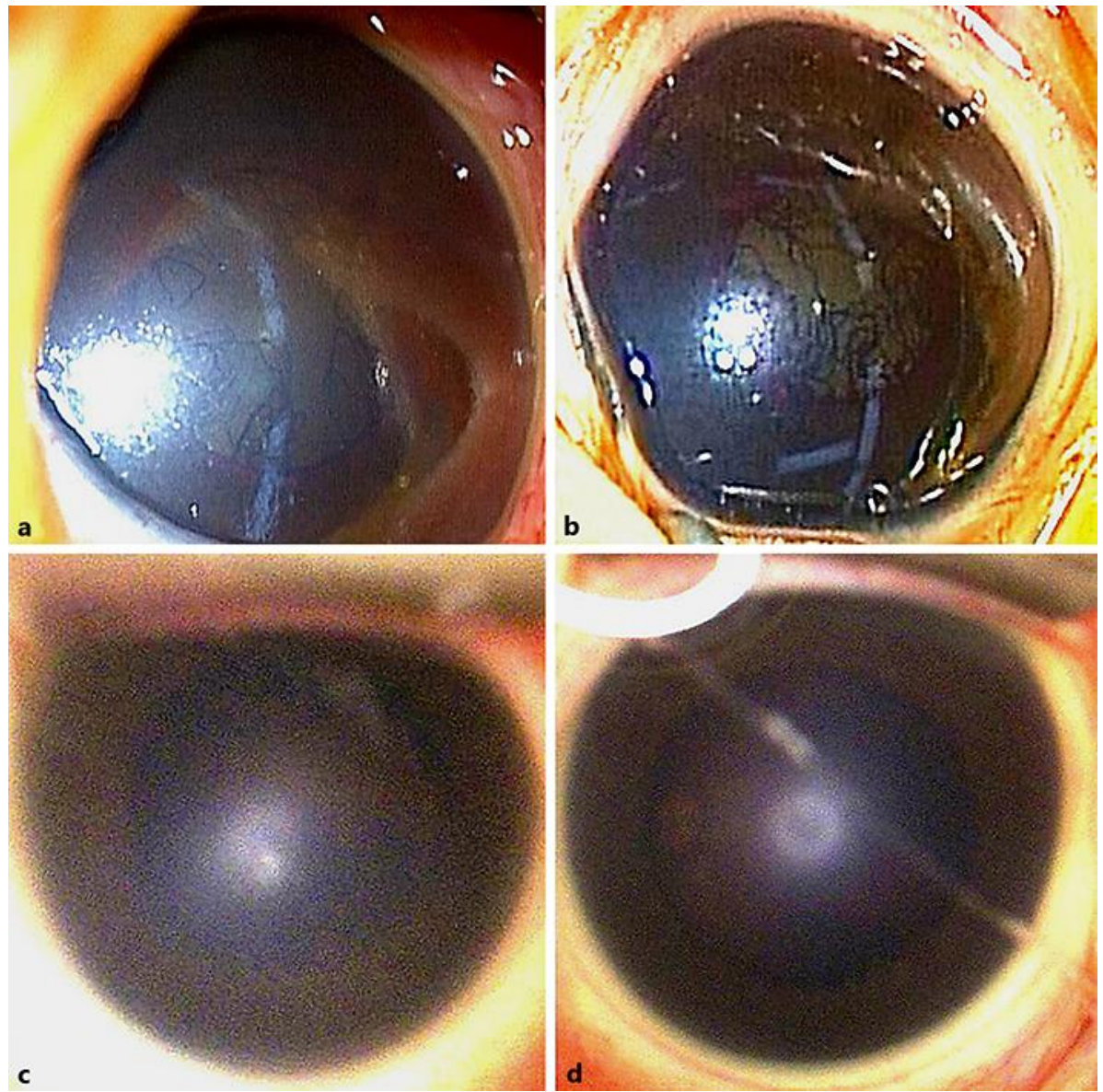

Fig. 3. Photographs of the anterior segment in case 2. a, b The anterior segment of the right eye (a) and the left eye (b) before treatment. Dilated tunica vasculosa lentis and poor mydriasis were observed in both eyes. c, $\mathbf{d}$ The anterior segment of the right eye (c) and the left eye (d) 3 days after treatment. The dilated tunica vasculosa lentis had regressed and mydriasis was improved in both eyes. 